\title{
Experimental validation of deep-subwavelength diffusion by acoustic metadiffusers
}

\author{
E. Ballestero, ${ }^{1, \text { a) N}}$ N. Jiménez, ${ }^{2}$ J.-P. Groby, ${ }^{3}$ S. Dance, ${ }^{1}$ H. Aygun, ${ }^{1}$ and V. Romero-García ${ }^{3}$ \\ 1) The Acoustics Group, London South Bank University, United Kingdom. \\ ${ }^{2)}$ Instituto de Instrumentación para Imagen Molecular, Consejo Superior de Investigaciones Científicas (CSIC), \\ Universitat Politècnica de València, València, Spain. \\ ${ }^{3)}$ Laboratoire d'Acoustique de l'Université du Mans, LAUM - UMR 6613 CNRS, Le Mans Université, Avenue Olivier Messiaen, \\ 72085 LE MANS CEDEX 9, France.
}

(Dated: 11 May 2019)

An acoustic metadiffuser is a subwavelength locally resonant surface relying on slow sound propagation and consisting of rigidly backed slotted panels, with each slit being loaded by an array of Helmholtz resonators (HRs). Due to the slow sound properties, the effective thickness of the panel can therefore be dramatically reduced when compared to traditional diffusers made of quarter-wavelength resonators. The aim of this work is to experimentally validate the concept of metadiffusers from the scattering measurements of a specific metadiffuser design, i.e., a Quadratic Residue Metadiffuser (QRM). The experimental results reported herein show a close agreement with analytical and numerical predictions, therefore showing the potentials of metadiffuser for controlling sound diffusion at very low frequencies.

PACS numbers: 43.20.Fn, 78.76.Pt

Scattering of waves is one of the most analysed phenomena in wave physics, and many applications using structures and surfaces that control the reflection of waves are exploited in several branches of science and technology. In acoustics, systems presenting a uniform scattering function, i.e., structures that reflect impinging waves in many different directions, have been largely developed since the pioneering work on acoustic diffusers by Schroeder in the $1970 \mathrm{~s}^{1}$. These locally-reacting surfaces spread reflected waves into all directions, reducing the strength of the undesired specular reflections and audible echoes while sound energy is preserved in space. The spatially-dependent reflectivity of a sound diffuser is generally tailored following numerical sequences with a uniform spatial Fourier transform of their reflection coefficient such as the Quadratic Residue (QR), Maximum Length (MLS), Primary Root (PR) or Index sequences ${ }^{2,3}$. Traditionally, these structures, also called Schroeder diffusers, are designed using rigidly-backed slotted panels where each well acts as a quarter-wavelength resonator (QWR). Therefore, these phasegrating diffusers become thick and heavy structures when designed to manage low-frequency waves, e.g., the typical thickness of a quadratic residue diffuser (QRD) is quarter of the wavelength corresponding to the low cut-off frequency. In this way, their application is very limited in critical listening environments such as auditoria, professional broadcast and recording control rooms, recording studios or conference rooms to control low-frequency sound.

Several approaches have been proposed in the past to overcome these limitations. Well folding strategies were proposed to minimize the unused space between slots ${ }^{4,5}$. Later, $\mathrm{Hu}-$ necke et.al. ${ }^{6}$ proposed to close the quarter wavelength resonators (QWRs) by perforated or microperforated sheets ${ }^{2,7}$, adding inertia to the impedance of the wells in order to lower the resonance frequencies and hence lower the design fre-

\footnotetext{
a)corresponding author: balleste@1sbu.ac.uk / ballesteroeric@outlook.com
}

quency. Recently, sonic crystals (SC) were used to construct volumetric acoustic diffusers ${ }^{8,9}$. In addition, optimized sound diffusers made of slotted panels incorporating two dimensional Helmholtz resonators (HRs) instead of QWRs are already commercialized ${ }^{10}$. By using HRs the resonance frequency of each well can be downshifted thus extending the diffusion bandwidth. This idea has recently been revisited by using metamaterials allowing the design of metasurfaces presenting simultaneously efficient diffusion properties and subwavelength dimensions. In 2017, Zhu et al. ${ }^{11}$ revisited the problem to design an ultra-thin QRD using a planar array of HRs. The main limitation of their approach is that the performance at high frequencies is reduced because wells are directly replaced by planar Helmholtz resonators. Also in 2017, the concept of metadiffusers was proposed by Jiménez et al. ${ }^{12}$. These sound diffusers are rigidly-backed slotted panels based on slow-sound metamaterials, i.e., each slit is loaded by an array of Helmholtz resonators. In essence, strong dispersion is introduced and the effective sound speed inside each slit is drastically reduced in the low frequency regime ${ }^{13,14}$ due to the loaded HRs. In this way, the quarter-wavelength resonance is shifted to the deep-subwavelength regime and, therefore, the effective thickness of the panel can be strongly reduced ${ }^{15-17}$. Various two-dimensional (2D) designs have been theoretically and numerically presented showing a strong reduction of the thickness of the panel of about a twentieth and a tenth of the thickness of traditional designs. As an example, a design of an optimized broadband metadiffuser panel of $3 \mathrm{~cm}$ thickness working from $250 \mathrm{~Hz}$ to $2 \mathrm{kHz}$ was presented ${ }^{12}$, i.e., the panel is then 24 times thinner that the lowest cut-off wavelength of the intended sequence.

In this work, we show for the first time the experimental validation of the concept of acoustic metadiffusers. We 3D printed a one-dimensional $\mathrm{QR}$ metadiffuser (QRM); meaning it only scatters sound efficiently in one plane; and experimentally characterized its diffusion properties in an anechoic chamber following the standard ISO 17497-2:2012 ${ }^{18}$. Note 

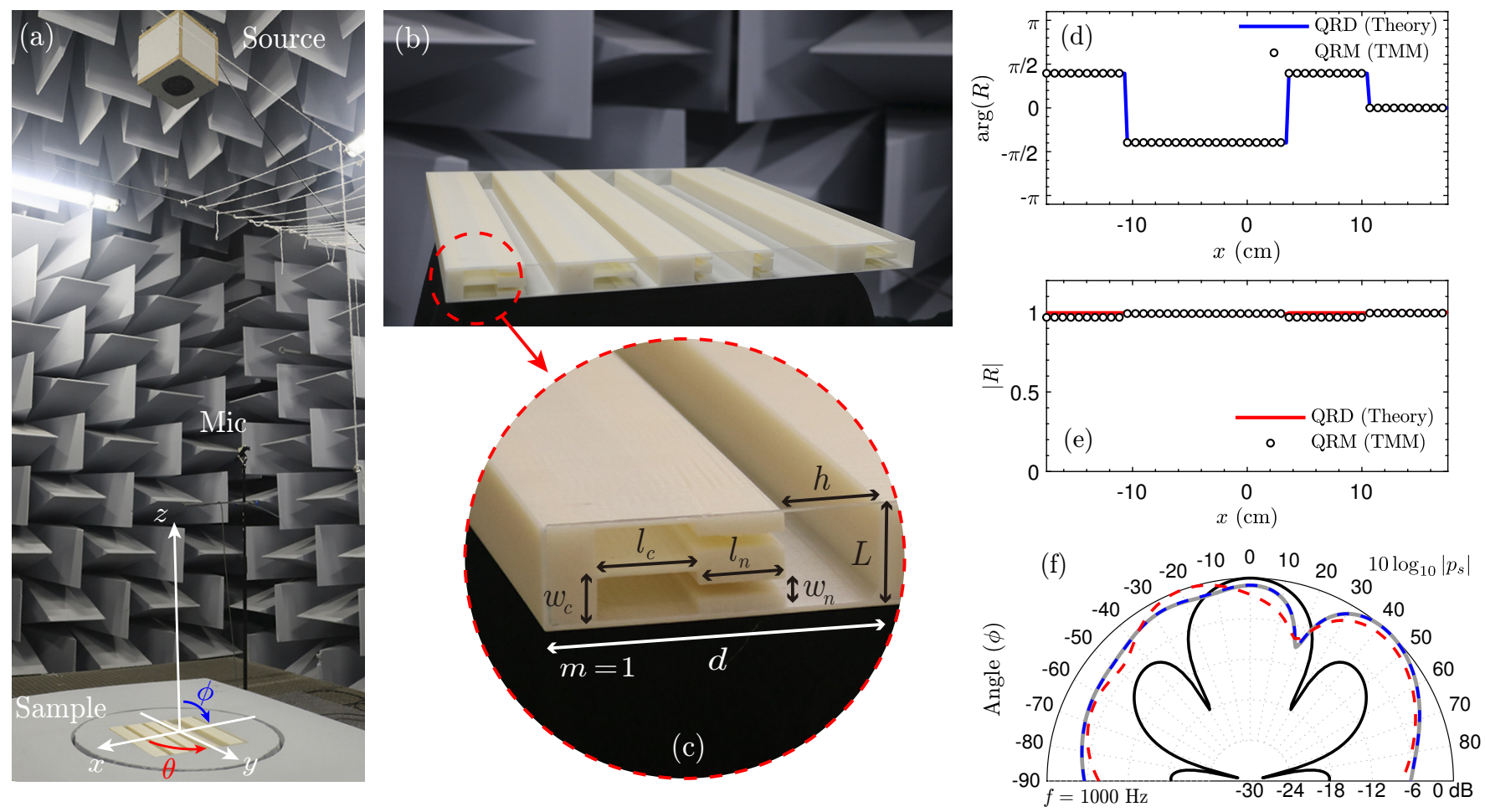

FIG. 1. (a) Experimental setup and coordinate system. (b) Photograph of the QRM, (c) detail of the first slit $(m=1)$ showing the definition of the geometrical parameters. (d) Phase and (e) magnitude of the spatially-dependent reflection coefficient for an ideal QRD (continuous lines) and the tailored QRM (markers). (f) Far-field polar distribution of the QRM obtained theoretically by TMM (dashed blue), numerically by FEM (dashed red), a ideal QRD (grey), and a plane reference reflector with same dimensions (continuous black) in (f), $f=2000 \mathrm{~Hz}, \mathrm{no}$.

that the original design ${ }^{12}$ was purely two-dimensional, however, in practice the structure must be bounded and the final structure thus becomes finite in the three dimensional space. The experimental results of the QRM and a reference flat rigid reflector are compared with 3D numerical predictions of the finite structures using the Finite Element method (FEM) including thermoviscous losses. As we will show, the experimental results reported herein are in close agreement with simulations and theory, therefore showing the potential of metadiffusers for controlling sound diffusion at the subwavelength scale.

The panel was manufactured using fused deposition modelling (FDM) techniques (Stratasys Fortus 450 MC), as shown in Figs. 1 (a-c). The squared panel, of $L=2 \mathrm{~cm}$ thickness and side $M d=35 \mathrm{~cm}$, is composed of $M=5$ slits and each slit is loaded with 2 identical HRs. This QRM mimics the behavior of a classical QRD made of $M$ wells and a total thickness of $L=27.4 \mathrm{~cm}$ designed for a low cut-off frequency of $500 \mathrm{~Hz}$. Note that the design frequency is normally set as the lower frequency limit of the diffuser, but it is not necessarily the lowest frequency at which the surface produces more scattering than a plane surface: the ratio between the size of the panel and the wavelength must be accounted for ${ }^{19}$. In this work, the response was evaluated at $2000 \mathrm{~Hz}$ to avoid the strong diffractive regime of the finite panel due to the small lateral size. The spatially-dependent reflection coefficient calculated from the QR sequence is given by $s_{m}=m^{2} \bmod (M)$, where $\bmod (M)$ is the least non-negative remainder of the prime number $M$.
For a classical QRD, the depth of the wells in the sequence is thus given by ${ }^{19,20} L_{m}=s_{m} \lambda_{0} / 2 M$, where $\lambda_{0}$ is the design wavelength. The dimensions of the slits and HRs of the QRM that mimics the reflection coefficient of the QRD are shown in Table I. The magnitude and phase of the reflection coefficient along the $x$-direction of the ideal QRD and the QRM respectively as calculated by using the transfer matrix method $(\mathrm{TMM})^{12}$ are shown in Figs. 1 (d, e). Here, the reflection coefficient is evaluated considering the viscothermal losses existing in both the QRD and the QRM ${ }^{21}$. Notice that the thickness of the QRM is reduced 13 times with respect to the classical QRD one while both responses match.

TABLE I. Geometrical parameters for the $m$-th well of the QRM. $h$ is the height of the slit, and $l_{n}, l_{c}, w_{n}$ and $w_{c}$ are the length and width of the neck and the cavity, respectively.

\begin{tabular}{ccccccc}
\hline$m$ & $s_{m}$ & $h(\mathrm{~mm})$ & $l_{n}(\mathrm{~mm})$ & $l_{c}(\mathrm{~mm})$ & $w_{n}(\mathrm{~mm})$ & $w_{c}(\mathrm{~mm})$ \\
\hline 1 & 1.0 & 14.7 & 13.0 & 16.4 & 6.2 & 9.0 \\
2 & 4.0 & 52.0 & 9.1 & 7.0 & 2.0 & 9.0 \\
3 & 4.0 & 30.9 & 9.1 & 4.3 & 3.5 & 9.0 \\
4 & 1.0 & 15.7 & 13.3 & 17.0 & 6.3 & 9.0 \\
5 & 0.0 & 20.3 & 18 & 20.7 & 3.2 & 9.0 \\
\hline
\end{tabular}

The far-field pressure distribution, $p_{s}(\theta, \phi)$, of a reflecting rectangular surface with a spatially dependent reflection coefficient, $R(x, y)$, of size $2 a$ and $2 b$ in the $x, y$-directions respec- 

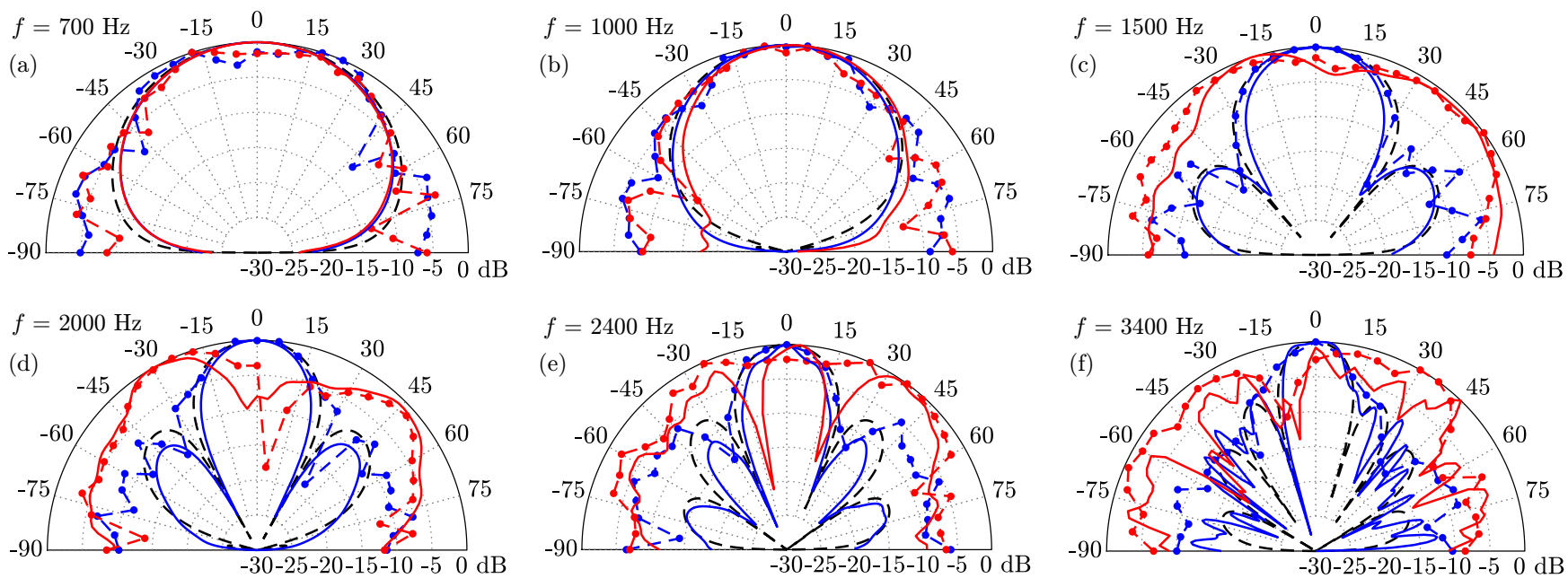

\begin{tabular}{|ll}
\hline Flat Pannel (Theoretical) & - Flat Pannel (Numerical) \\
- - Flat Pannel (Experiment)
\end{tabular}

- Metadiffuser (Numerical)

- - Metadiffuser (Experiment)

FIG. 2. Scattered field distribution, $p_{s}\left(90^{\circ}, \phi\right)$, at different frequencies obtained experimentally in the near-field (markers) and numerically (continuous) for the QRM (red) and the flat reference panel (blue) in the far-field. Theoretical far-field results for the flat panel are shown in black-dashed lines. Change lines of flat panel experimental to only dashed lines (not dot). Legend: theoretical should be dashed.

tively, can be calculated using the Fraunhofer integral ${ }^{19}$

$$
p_{s}(\theta, \phi)=\int_{-a}^{a} \int_{-b}^{b} R(x, y) e^{i k x \sin \phi \cos \theta} e^{i k y \sin \phi \sin \theta} \mathrm{d} x \mathrm{~d} y,
$$

with $\theta$ and $\phi$ being the reflected azimuthal and elevational polar angles respectively, as shown in Fig. 1 (a). Figure 1 (f) shows the far-field calculations in the $x$-varying plane at 2000 $\mathrm{Hz}$ for a $\mathrm{QRD}$, the QRM and a flat reference reflector of same dimensions. Excellent agreement is observed between the polar responses using the direct application of the Eq. (1) and using a reflection coefficient calculated using TMM and from the full-wave numerical solution using the FEM (see further details of the methods in Jiménez et al. ${ }^{12}$ ). Note viscothermal losses were accounted for in both cases.

An experimental procedure to evaluate the diffusion of such kind of diffusers following the measurement standard ISO 17497-2:2012 $2^{18}$ was developed to determine the sound scattering properties of the QRM. As such, measurements consisted in placing the physical sample (e.g., the QRM or the flat reference panel) at the centre of a virtual concentric arc of evenly-spaced microphone positions, all within an anechoic environment and keeping unwanted acoustical contributions from the measurement system as minimal as possible, as shown in Fig. 1 (a). Microphone positions ranged from $\phi=0^{\circ}$ to $\phi=90^{\circ}$ around the surface with a spacing of $6^{\circ}$ between each and along an arc radius of $1 \mathrm{~m}$. The sample was placed on a rotating table, thus allowing a complete hemispherical characterization of the surface's scattering along the polar circle in the $\theta$ direction. Measurements were performed for normal incidence by locating the source $2.5 \mathrm{~m}$ away from the surface. The system was excited using a broadband Maximum Length Sequence (MLS) signal and impulse responses
(IRs) at each microphone position were obtained by deconvolution. Each IR was subtracted to the one obtained from the anechoic background and windowed to extract the scattered sound field ${ }^{18}$. Then the polar distribution of the scattered field was obtained after Fourier transformation.

The experimental, analytical and simulated scattered field distributions at $\theta=90^{\circ}$ cross-section are shown in Fig. 2 for both the QRM and the flat reference panel. Analytical solutions of Eq. (1) for a finite rigid panel of dimensions $a=b=35 \mathrm{~cm}$ can be found in the literature ${ }^{22}$. Here, we show frequencies ranging from $700 \mathrm{~Hz}$ to $3.4 \mathrm{kHz}$. On the one hand, the simulated scattered field of the flat panel agrees with the analytical one (continuous-blue and dashed-black, respectively). Slight deviations are observed at $2.4 \mathrm{kHz}$ and $3.4 \mathrm{kHz}$, mainly caused by the finite thinness of the panel in the simulation. The measured scattered field of the flat panel (dashed blue) also shows a strong agreement with the simulated and theoretical ones, except at grazing angles $\left(\phi>60^{\circ}\right)$ where higher scattering values are observed. This occurs because the weak reflected energy by the panel at grazing angle is comparable to the spurious reflections of the anechoic chamber grid that covers the floor. However, this effect has a very low impact on the diffusion coefficient values, as we will see later. On the other hand, measured scattered field values for the QRM (dashed red + markers) are in close agreement to the far-field ones (red continuous) obtained through FEM simulations. Note the dips observed in the simulations are smoothed in the experimental data and the overall distribution shape is conserved. At low frequencies, e.g., $700 \mathrm{~Hz}$, simulated curves for the flat panel and QRM illustrate the fact that the QRM behaves in a similar manner to that of the flat panel, showing the need of normalization to estimate the diffusion performance of the sample. 

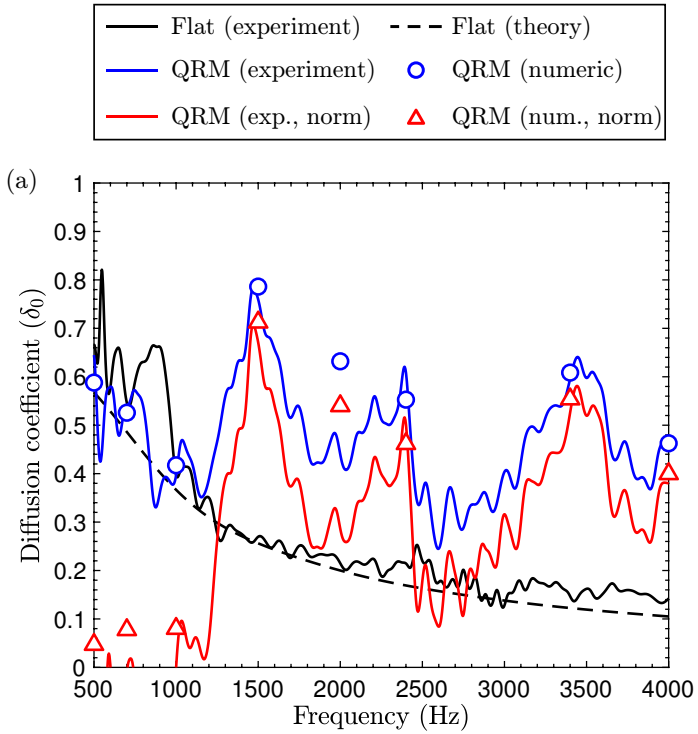

(b)

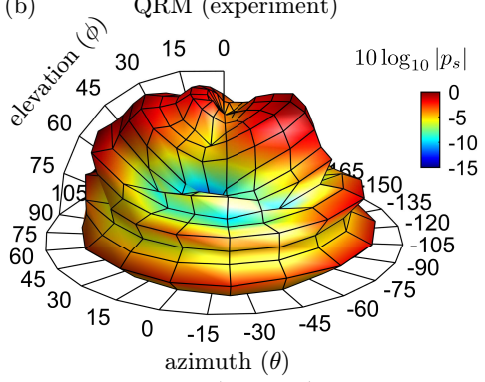

(c)

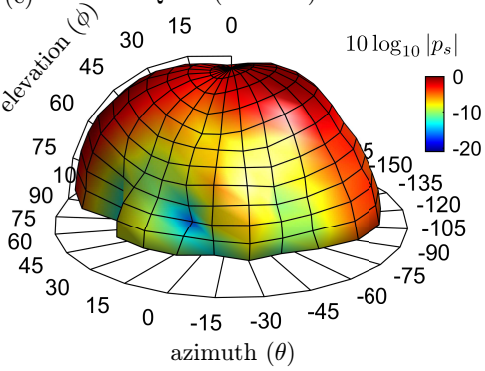

(d) Flat reference (experiment)

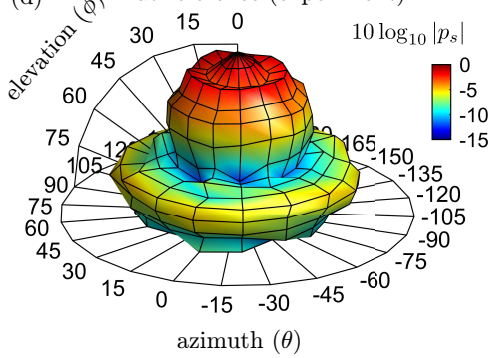

(e) Flat reference (theory)

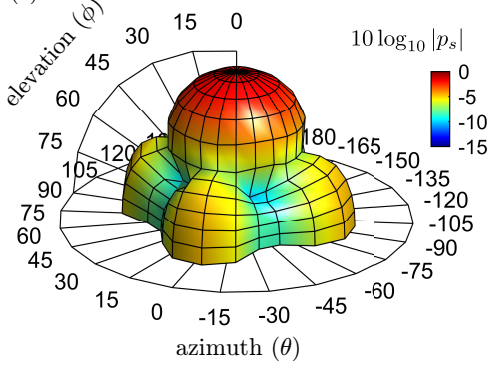

FIG. 3. (a) Diffusion and normalized coefficients of the QRM and a flat reference panel. Scattered field distribution, $p_{s}(\theta, \phi)$, for the QRM at $1500 \mathrm{~Hz}$ (b) measured experimentally and (c) by FEM simulations. (d-e) Corresponding scattered field distribution for the flat reference panel. V: Eric, could you add more points in Fig. 3(a)? E: QRM NUM and FLAT REF seem glitched (cut near 60 phi), see EPS rendering maybe?

Ultimately, the directional diffusion coefficient ${ }^{23}$ produced when the diffuser is radiated by a plane wave at the incident angles $\left(\theta^{\prime}, \phi^{\prime}\right), \delta_{\theta^{\prime}, \phi^{\prime}}$, can be estimated from the hemispherical distributions as

$$
\delta_{\theta^{\prime}, \phi^{\prime}}=\frac{\left[\iint I_{S}(\theta, \phi) d S\right]^{2}-\iint I_{s}^{2}(\theta, \phi) d S}{\iint I_{S}^{2}(\theta, \phi) d S},
$$

where $I_{s}(\theta, \phi)=\left|p_{s}(\theta, \phi)\right|^{2}$ is proportional to the scattered intensity. The integration is performed over a hemispherical surface $(-\pi / 2 \leq \phi \leq \pi / 2$ and $\theta=2 \pi)$ where $d S=d \theta d \phi$. In this work we analyse the case of a normal incident wave, i.e., $\theta^{\prime}=0$ and $\phi^{\prime}=0$. Therefore, $\delta_{\theta^{\prime}, \phi^{\prime}} \equiv \delta_{0}$. This coefficient must be normalized to that of a plane reflector, $\delta_{\text {flat }}$, to eliminate the effect of the finite size of the structure as $\delta_{n}=\left(\delta_{0}-\delta_{\text {flat }}\right) /\left(1-\delta_{\text {flat }}\right)$. Then, $\delta_{n}$ tneds to 0 when the sample scatters energy with a similar polar distribution as the reference reflector, while $\delta_{n}$ tends to 1 when the waves reflected by the sample are scattered evenly in all directions in a far more omnidirectional way than the flat reference reflector. Thus, the frequency-dependent performance of a diffuser can be evaluated from the directional of the normalized diffusion coefficient value.

Figure 3 (a) shows the frequency-dependent directional diffusion coefficients, $\delta_{0}$, calculated analytically, numerically and experimentally for the QRM and the flat panel. First, the experimental diffusion coefficient for the flat panel is in close agreement with the analytical one and, as expected, higher diffusion values are achieved in the low frequency regime due to the diffraction of the finite sample. The same phenomenon can be observed for the QRM (blue continuous) in this low frequency regime as it matches values obtained for the flat panel. This is mainly due to the lack of any slit resonance within the metasurface, and is thus illustrated by the normalized diffusion coefficient of the QRM taking values around zero. However, when approaching the critical slit resonance at $f=1500 \mathrm{~Hz}$ the dispersion in the slits progressively changes and the impedance of each deep-subwavelength slit is hence modified to match the impedance sequence of the tailored QRD. The complex reflection coefficient becomes spatially dependent and, then, following Eq. (1) the scattering distribution start to be modified. Eventually the spatially-dependent reflection coefficient matches the one of a QRD at $f=1500$ $\mathrm{Hz}$. At this frequency, the experimental diffusion coefficient takes a value of $\delta_{0}=0.783$, while the corresponding simulated one is placed at a very close value of $\delta_{0}=0.786$. The normalized diffusion coefficient takes a value of $\delta_{n}=0.708$ in the experiment and $\delta_{n}=0.712$ in the simulation, keeping these values in the range of those reported for classical QRDs ${ }^{19}$. Note the normalized diffusion coefficient using 1D theory (see Fig.1(f)) takes a close value $\left(\delta_{n}=0.69\right)$. However, the latter 1D diffusion value must not be directly compared with results in Fig.3 (a) as oblique and transversal modes along the $y$-direction are not included in the 1D theory. The presence of such modes will affect the impedance of the slits and will thus result in a change of the scattering properties of the surface. The experimental and simulated and theoretical scattering distributions at $f=1500 \mathrm{~Hz}$ at a distance of $1 \mathrm{~m}$ from the sample are shown in Figs. 3 (b-e). For the QRM the waves are reflected evenly for the azimuthal plane, $\theta= \pm 90^{\circ}$, corresponding to the cross-section of the slits as $R(x, y)$ only shows variations in the $x$-direction. This is an expected behaviour observed in any 1D phase grating diffuser and particularity useful to anisotropically control reflections in critical listening spaces ${ }^{19}$. In contrast, the flat panel mainly 
scatters waves in the specular direction. Both, experimental and numerical scattering distributions agree away from grazing angles as explained above. Complementary 3D plots at other frequencies and animated videos illustrating the simulated behavior of the QRM and flat panel are available in the supplementary material.

We have experimentally demonstrated the efficiency of metadiffusers, i.e., deep-subwavelength metasurfaces with uniform scattering distribution to scatter the acoustic energy in the subwavelength regime. The scattering distributions observed experimentally using 3D panels are in close agreement with simulated ones, and agree to the theoretical designs ${ }^{12}$. A remarkable high diffusion performance is demonstrated by the experimental normalized diffusion coefficient of $\delta_{n}>0.7$ at $1500 \mathrm{~Hz}$. The results shown herein demonstrate the possibility of metadiffusers to be applied in many practical situations where the lack of space to install classical solutions is limited. This includes applications ranging from opera pits ${ }^{2}$ to aerospace applications ${ }^{24}$. This study allows to push forth towards situation-specific designs of optimized metadiffusers and to continue measuring their scattering characteristics in order to solidify the knowledge of such subwavelength metasurfaces.

\section{ACKNOWLEDGMENTS}

This article is based upon work from COST Action DENORMS CA15125, supported by COST (European Cooperation in Science and Technology). This study was financed by the Royal Opera House, Covent Garden, and the United Kingdom Acoustics Network (UKAN). The authors gratefully acknowledge the ANR-RGC METARoom (ANR-18-CE080021) project and the project HYPERMETA funded under the program Étoiles Montantes of the Région Pays de la Loire. N.J. acknowledges financial support from Generalitat Valenciana through grant APOSTD/2017/042.

${ }^{1}$ M. R. Schroeder, The Journal of the Acoustical Society of America 57, 149 (1975).

${ }^{2}$ T. Cox and P. D'Antonio, Building Acoustics 10, 1 (2003).

${ }^{3}$ T. J. Cox and P. D'Antonio, "Acoustic absorbers and diffusers: Theory, design and application," (Crc Press, 2009) pp. 295-306.

${ }^{4}$ F. Mechel, Acta Acustica united with Acustica 81, 379 (1995).

${ }^{5}$ J. Hargreaves and T. Cox, Proceedings of the Institute of Acoustics 25, 199 (2003).

${ }^{6} \mathrm{~J}$. Hunecke, Schallstreuung und Schallabsorption von Oberf: ahen aus mikroperforierten Streifen, Ph.D. thesis, University of Stuttgart (1997).

${ }^{7}$ T. Wu, T. J. Cox, and Y. Lam, The Journal of the Acoustical Society of America 110, 3064 (2001).

${ }^{8}$ R. J. Hughes, J. A. Angus, T. J. Cox, O. Umnova, G. A. Gehring, M. Pogson, and D. M. Whittaker, The Journal of the Acoustical Society of America 128, 2847 (2010).

${ }^{9}$ J. Redondo, R. Picó, V. J. Sánchez-Morcillo, and W. Woszczyk, The Journal of the Acoustical Society of America 134, 4412 (2013).

${ }^{10}$ P. DAntonio, "Planar binary amplitude diffusor," (1998).

${ }^{11}$ Y. Zhu, X. Fan, B. Liang, J. Cheng, and Y. Jing, Physical Review X 7, 021034 (2017).

${ }^{12}$ N. Jiménez, T. J. Cox, V. Romero-García, and J.-P. Groby, Scientific Reports 7, 5389 (2017).

${ }^{13}$ J.-P. Groby, W. Huang, A. Lardeau, and Y. Aurégan, Journal of Applied Physics 117, 124903 (2015).

${ }^{14}$ A. Santillán and S. I. Bozhevolnyi, Phys. Rev. B 84, 064304 (2011).
${ }^{15}$ N. Jiménez, W. Huang, V. Romero-García, V. Pagneux, and J.-P. Groby, Applied Physics Letters 109, 121902 (2016).

${ }^{16}$ N. Jiménez, V. Romero-García, V. Pagneux, and J.-P. Groby, Physical Review B 95 (2016), 10.1103/PhysRevB.95.014205.

${ }^{17}$ N. Jiménez, V. Romero-García, V. Pagneux, and J.-P. Groby, Scientific Reports 7, 13595 (2017).

${ }^{18}$ I. S. Organization, "ISO 17497-2:2012 Acoustics - Sound-scattering properties of surfaces - part 2: Measurement of the directional diffusion coefficient in a free field," (2012).

${ }^{19}$ T. J. Cox and P. D'Antonio, Acoustic absorbers and diffusers: theory, design and application, 3rd ed. (CRC Press, 2016).

${ }^{20}$ T. Cox and P. D'Antonio, Building Acoustics 10, 1 (2003).

${ }^{21}$ M. R. Stinson, J. Acoust. Soc. Am. 89, 550 (1991).

${ }^{22}$ T. D. Rossing, F. Dunn, W. M. Hartmann, D. M. Campbell, and N. H. Fletcher, Springer Handbook of Acoustics, 1st ed. (Springer Publishing Company, Incorporated, 2007).

${ }^{23}$ ISO 17497-2:2012, "Acoustics - Sound-scattering properties of surfaces Part 2: Measurement of the directional diffusion coefficient in a free field," ISO Standard (International Organization for Standardization, Geneva, Switzerland, 2012).

${ }^{24}$ L. Garcia-Raffi, L. Salmeron-Contreras, I. Herrero-Durá, R. Picó, J. Redondo, V. Sánchez-Morcillo, K. Staliunas, N. Adkins, A. Cebrecos, N. Jiménez, et al., Aerospace Science and Technology 73, 300 (2018). 